\title{
Modelo de oficinas de qualificação em Aprendizagem Baseada em Equipes com docentes de Medicina
}

\section{A model for qualification workshops in team-based learning with medicine teachers}

\author{
Mário Roberto Tavares Cardoso de Albuquerque ${ }^{1,2}$ (D) $\mid$ drmarioalbuquerque@gmail.com \\ Nara Macedo Botelho ${ }^{2}$ (D) narambotelho@gmail.com \\ Milena Coelho Fernandes Caldato ${ }^{1,2}$ (1) milenacaldato@cesupa.br
}

\section{RESUMO}

Introdução: A Aprendizagem Baseada em Equipes ( $\mathrm{ABE}$ ) tem se mostrado, em todo o mundo, uma ferramenta pedagógica promissora nas mais diversas realidades, ao estimular o autoaprendizado do aluno e potencializar a habilidade de trabalhar em equipe. Todavia, são escassos os estudos que envolvam a qualificação de docentes para a prática dessas atividades.

Objetivo: Dessa forma, o presente estudo objetivou relatar um modelo de oficina de qualificação docente em ABE e a avaliação feita pelos participantes acerca da metodologia empregada, com docentes do curso de Medicina de uma instituição privada de ensino superior de Belém, no Pará.

Método: Trata-se de uma pesquisa-ação intervencionista realizada por meio de questionários elaborados pelos próprios dos autores para avaliar a satisfação, as críticas e as recomendações dos docentes acerca das oficinas propostas.

Resultados: Percebeu-se que $81,2 \%$ dos participantes atribuíram "satisfação máxima" às oficinas e os demais as classificaram como "satisfatórias". Dessa forma, houve aprovação da técnica empregada por todos os docentes. Quando indagados sobre os pontos negativos nas oficinas, os mais citados foram: horários e dias escolhidos (18,7\%); pouco tempo para estudo individual (15,6\%); deslocamento, carga horária extensa e falhas no convite para inscrição nas oficinas (12,5\%). Quando questionados sobre os pontos positivos, os mais citados foram: utilizar a própria $A B E$ para ensinar ABE (93,7\%); qualidade dos artigos escolhidos para estudo prévio (87,5\%); e alta aplicabilidade prática (81,2\%). Por fim, 93,7\% dos participantes referiram se sentir confiantes para conduzir atividades de ABE em suas práticas diárias.

Conclusão: Os participantes demonstraram boa aceitação e satisfação com relação à metodologia empregada nas oficinas, permitindo assim que possam aplicar essa estratégia de ensino com maior segurança e assertividade em suas rotinas diárias. Espera-se que este trabalho possa contribuir para a aprendizagem sobre essa estratégia educacional e descomplicar e tornar acessível a sua aplicação, para assim estimular docentes e instituições não familiarizados com a ABE a introduzir essa valiosa ferramenta em suas matrizes pedagógicas.

Palavras-chave: Autoaprendizagem como Assunto; Educação Médica; Docentes de Medicina.

\section{ABSTRACT}

Introduction: Team-Based Learning (TBL) has proven to be a promising pedagogical tool in the most diverse realities around the world, stimulating student self-learning and enhancing the ability to work in teams. However, there are few studies involving the qualification of teachers to practice these activities.

Objective: Thus, the present study aimed to report a model of a teacher qualification workshop in TBL and the evaluation made by the participants about the methodology used, with teachers of the medical course of a private higher education institution in Belém/PA/Brazil.

Method: This was an interventionist action research conducted through questionnaires by the authors to assess teacher satisfaction, criticisms and recommendations about the proposed workshops.

Results: It was found that $81.2 \%$ of the participants rated the workshops as providing "maximum satisfaction" and the rest classified them as "satisfactory". Thus, all the teachers approved of the technique used. When asked about the negative points in the workshops, the most frequently cited were: selected times and days (18.7\%); little time for individual study (15.6\%); and commuting, extensive workload and failures in the invitation to enroll in the workshops (12.5\% each item). On the other hand, when asked about the positive points, the most frequently cited were: using the TBL itself to teach TBL (93.7\%); quality of the articles chosen for previous study (87.5\%); and high practical applicability (81.2\%). Finally, 93.7\% of the participants reported feeling confident to conduct TBL activities in their daily practices.

Conclusion: In this and the vast majority of studies with TBL, the participants demonstrated good acceptance and satisfaction regarding the methodology used in the workshops, thus allowing them to apply this teaching strategy with greater security and assertiveness in their daily routines. It is hoped that this work can contribute to learning about this educational strategy and that it can simplify its application and enhance its accessibility, in order to stimulate teachers and institutions unfamiliar with TBL to introduce this valuable tool in their activities.

Keywords: Active Learning; Medical Education; Medical Faculty.

${ }^{1}$ Centro Universitário do Estado do Pará, Belém, Pará, Brasil.

2 Universidade do Estado do Pará, Belém, Pará, Brasil.

Editora-chefe: Daniela Chiesa

Editor associado: Kristopherson Lustosa Augusto

Recebido em 13/08/20; Aceito em 05/04/21.

Avaliado pelo processo de double blind review. 


\section{INTRODUÇÃO}

A Aprendizagem Baseada em Equipes - ABE (TeamBased Learning - TBL) tem se mostrado, em todos o mundo, uma ferramenta pedagógica promissora nas mais diversas realidades, ao estimular o autoaprendizado do aluno e potencializar a habilidade de trabalhar em equipe ${ }^{1-4}$.

Mas o que é a $A B E$ e o que a torna diferente das demais metodologias? Em síntese, essa estratégia educacional é constituída por um conjunto de práticas sequenciadas de ensino-aprendizagem que visa promover o desenvolvimento de equipes de aprendizagem de alto desempenho e fornecerIhes oportunidades para que possam se envolver em tarefas de aprendizagem significativas $s^{5-7}$.

Os princípios fundamentais da ABE são: 1. os grupos de aprendizado devem ser formados e gerenciados; 2 . os alunos devem ser responsabilizados pela qualidade do trabalho individual e do grupo; 3. eles devem receber feedback frequente e oportuno sobre a aprendizagem obtida; e 4. as atribuições da equipe devem promover a aprendizagem do conteúdo e o próprio desenvolvimento ${ }^{8}$.

A primeira etapa da metodologia consiste no preparo. Antes de cada módulo, os alunos devem realizar uma atividade dirigida que tem por objetivo a contextualização da temática e o embasamento para as questões a serem exploradas individualmente e pelas equipes (por exemplo: textos, vídeos, entrevistas, atividades práticas, entre outros). Quando se tratar de leituras, elas devem ser objetivas, não muito extensas e com tempo hábil para execução $0^{5,7}$. Trabalhos já realizados identificaram que a desproporção entre a quantidade de textos prévios e o tempo fornecido para estudo influencia na satisfação dos participantes com a $A B E^{9,10}$.

A segunda etapa é a garantia do preparo (GP). Essa fase inclui a realização de um mesmo teste, inicialmente de forma individual e depois em equipe, seguida por feedback, apelação e breve apresentação do professor. A duração dessa etapa varia de 30 a 60 minutos $^{11}$ ou de 45 a 75 minutos $^{7}$.

Parmelee et al. ${ }^{11}$ descrevem cinco objetivos principais dessa etapa: 1. abrangência do conteúdo; 2 . desenvolvimento das equipes; 3. valorização de múltiplas fontes; 4. desenvolvimento de habilidades de estudo; e 5. otimização do tempo de aula. Esse processo estimula o ensino-aprendizagem entre os pares, assim como feedback individual e para a equipe.

Inicialmente, o estudante realiza um teste individual de garantia de preparo (Individual Readiness Assurance Test - iRAT) e entrega o gabarito ao professor. Em seguida, o estudante realiza o mesmo teste, dessa vez com a sua equipe (Team Readiness Assurance Test - tRAT) ${ }^{11}$. Durante a realização do tRAT, os membros da equipe expõem e justificam suas escolhas, buscando um consenso sobre a resposta correta.
Esse processo estimula os estudantes a desenvolver habilidades de comunicação e negociação, assim como propicia o compartilhamento de conhecimentos e favorece o reconhecimento das potencialidades e fragilidades individuais, com a consequente conscientização do aluno sobre como ampliar sua participação e contribuição com a equipe ${ }^{5,6}$.

A terceira e última etapa consiste na aplicação dos conceitos. Para Michaelsen ${ }^{7}$, essa fase é a mais importante para a consolidação dos conhecimentos e pode durar de uma a quatro horas. Ela envolve um conjunto de exercícios práticos que permite aos estudantes aprofundar os conceitos aprendidos por meio do trabalho em equipe na resolução de questões e problemas.

Os exercícios devem apresentar situações e cenários semelhantes aos que os estudantes vivenciarão ao longo de sua formação e vida profissional e devem desafiá-los a fazer interpretações, cálculos, comparações, previsões, análises, avaliações e sínteses das informações, visando à escolha de uma opção ou resposta pela equipe $\mathrm{e}^{5-7}$.

Os autores de uma revisão sistemática recente ${ }^{12}$ avaliaram a literatura publicada e considerada relevante sobre ABE. Eles constataram que $72 \%$ dos artigos incluíam a avaliação de resultados de aprendizagem e que a maioria dos estudos envolvia alunos de graduação. Desses artigos, $61 \%$ concluíram que a $A B E$ era uma técnica instrucional eficaz. Contudo, ainda são escassos trabalhos que abordem os docentes nessas atividades e, principalmente, a forma de qualificá-los para que possam ser instrutores de $A B E$.

\section{MÉTODO}

A pesquisa foi submetida ao Comitê de Ética em Pesquisa (CEP), tendo sido aprovada conforme Parecer $\mathrm{n}^{\circ}$ 3.054.944. Os sujeitos selecionados tiveram suas identidades totalmente resguardadas, respeitando a Resolução n 466/2012 do Conselho Nacional de Saúde (CNS), seguindo os preceitos da Declaração de Helsinki e do Código de Nuremberg.

Trata-se de uma pesquisa-ação intervencionista ${ }^{13}$ que supõe intervenção participativa na realidade social estudada, de abordagem longitudinal e prospectiva, por meio de oficinas de qualificação docente. Realizou-se o estudo com docentes de Medicina de uma instituição de ensino superior (IES) privada de Belém, no Pará. A casuística foi determinada por livre demanda, tendo sido ofertadas 50 vagas para duas oficinas sobre $A B E$ dentro da semana de desenvolvimento docente da instituição. Consideraram-se o espaço e os recursos para sua realização, em que 47 docentes se inscreveram e 32 participaram das oficinas. A convocação foi feita por endereço eletrônico com inscrições pelo Google Forms.

Os professores foram convidados a participar de duas 
oficinas de capacitação na metodologia da ABE que ocorreram em dois momentos distintos com uma semana de intervalo entre as etapas. A primeira etapa foi comum às duas oficinais, sendo feita uma explanação sobre os objetivos e componentes da atividade, realizada pelo autor principal do estudo com utilização de recursos audiovisuais a exemplo de apresentações em PowerPoint.

Na primeira oficina, realizou-se a apresentação do estudo aos docentes, entregaram-se os Termos de Consentimento Livre e Esclarecido (TCLE), e aplicou-se um questionário com oito perguntas fechadas, com a finalidade de avaliar o conhecimento prévio dos docentes sobre a $A B E$ e definir o perfil profissional dos participantes. Essa etapa teve duração aproximada de 30 minutos. É importante destacar que os questionários não foram identificados com os nomes dos participantes, a fim de não comprometer o sigilo das informações e nem possíveis conflitos de interesse por parte dos autores. Outros dados da ficha protocolar incluíam idade, sexo, titulação e anos de atuação na docência, que serviram posteriormente para equiparar os membros das equipes.

A disposição da sala de aula para as oficinas seguiu o proposto pela nota técnica de Lima et al. ${ }^{14}$. Para os autores, o melhor formato da sala deve distribuir as mesas de tal modo que todos consigam ver projeções de multimídia de seus respectivos lugares. Se o espaço não permitir essa disposição, outros arranjos poderão ser feitos, desde que, no momento da projeção, os participantes direcionem suas cadeiras para o painel de multimídia. Além de mesas e cadeiras, deve haver uma mesa e um espaço adequado para o facilitador apoiar o material didático, preferencialmente perto do painel de multimídia.

Ainda nessa oficina, após a resolução do questionário de avaliação do conhecimento prévio dos docentes, realizou-se uma breve explanação em PowerPoint ${ }^{\circledR}$ pelo autor acerca das etapas e propostas da $\mathrm{ABE}$, com duração de aproximadamente 30 minutos. Após a apresentação, fez-se uma roda de conversa com o propósito de analisar as dificuldades encontradas, as opiniões e as sugestões dos professores para implementar a $\mathrm{ABE}$ em suas práticas diárias, com duração de 60 minutos, totalizando duas horas para realização da oficina 1.

Ao final, entregaram-se aos participantes dois artigos de autoria de Silva Junior et al. ${ }^{15}$ e Krug et al. ${ }^{6}$ para estudo dirigido, os quais abordam a metodologia preconizada para realização da $A B E$ e seus aspectos teóricos. Sugeriu-se consulta ao site da organização Team-Based Learning Collaborative ${ }^{16}$ que tem grande utilidade para quem deseja obter um aprofundamento sobre o tema, assim como fornece referências de artigos que detalham sobre como delinear as tarefas e atividades em cada uma das etapas. Além disso, serviu de base para as atividades da oficina seguinte que ocorreu uma semana depois, no mesmo local e horário.
$\mathrm{Na}$ segunda oficina, foi feita a qualificação sobre a metodologia da $A B E$ após leitura prévia dos artigos entregues pelo autor (etapa de preparo prévio, conforme preconizado pela metodologia da ferramenta pedagógica), utilizando as próprias etapas da ABE. Entregou-se aos participantes um teste de garantia do preparo (TGP) individual com oito questões de múltipla escolha, contendo quatro alternativas, com cerca de 20 minutos para resolução.

Em seguida, os docentes foram distribuídos em quatro grupos de oito participantes, de forma intencional, randomizada e equiparada conforme anos de experiência na docência e experiência em $A B E$, além de sexo, idade e titulação.

Na próxima etapa, ocorreu a resolução do TGP em grupo com duração estimada de 20 minutos. Na sequência, o autor da pesquisa exibiu as questões para todos os grupos em slides e foram entregues quatro placas com alternativas de " $A$ " $a$ " $D$ ", para que cada grupo defendesse a sua resposta, gerando discussão e feedback imediato pelo autor da pesquisa, com cerca de 30 minutos de duração.

Para o somatório dos pontos no TGP, escolheu-se o sistema de pontuação de Michaelsen, em que cada questão tem um valor máximo de quatro pontos. Ao final, realizou-se o somatório dos pontos que poderia atingir um máximo de 32 pontos. Posteriormente, iniciou-se a arguição entre os grupos, em que cada um levantava, ao mesmo tempo, uma placa com a alternativa escolhida, e, no caso de respostas divergentes, cada grupo deveria defender a escolha feita. Essa etapa demandou mais 30 minutos para realização, totalizando 70 minutos para execução da segunda etapa, dentro do preconizado pela metodologia.

Na terceira e última etapa, foi entregue aos participantes um caso complexo, com situação realística envolvendo a implementação da $A B E$ no ensino superior, com 60 minutos para realização. Em seguida, todos os grupos propuseram sugestões. Esse processo serviu para que cada um pudesse implementá-las em suas práticas diárias, nas quais a metodologia ainda não é empregada. Cada grupo teve que apresentar um planejamento estratégico em cartolina com pontos críticos e possíveis soluções para a problemática proposta, aplicando, dessa forma, os conceitos teóricos estudados em uma vivência prática, como defende a metodologia da ABE.

Por fim, entregaram-se os questionários finais de avaliação das oficinas, com a finalidade de verificar pontos a serem melhorados em futuras iniciativas. Para avaliar a satisfação, foi utilizada uma escala Likert de 1 a 5 (1 = "muito insatisfatórias" e $5=$ "muito satisfatórias"). O protocolo de pesquisa objetivou avaliar o grau de satisfação dos participantes, os pontos positivos a serem aperfeiçoados dos modelos de oficinas propostos, além da sensibilização dos docentes com relação às vantagens da $A B E$ e à confiança 
em implementá-la em suas práticas diárias. A oficina 2 foi realizada em duas horas e 30 minutos.

Após a coleta de dados, elaborou-se uma planilha eletrônica para armazenamento dos dados, no software Microsoft Excel 365. Para representação descritiva dos dados, elaboraram-se tabelas com a utilização do software Microsoft Word 365. As frequências absolutas e relativas foram descritas para as variáveis categóricas. Para o processamento da análise dos dados, utilizou-se o programa SPSS versão 23. A fim de comparar as concordâncias e discordâncias entre os pares de observações nos dois momentos (antes e depois da oficina de $A B E)$ sobre a sensibilização dos docentes acerca dos benefícios dessa estratégia educacional, utilizou-se o teste de McNemar, considerando um nível de significância de $5 \%(p<0,05)$.

\section{RESULTADOS}

Constatou-se que $81,2 \%$ dos participantes atribuíram "satisfação máxima" às oficinas e os demais as classificaram como "satisfatórias" (Tabela 1). Dessa forma, houve aprovação da técnica empregada por todos os docentes.

Quando indagados sobre os pontos negativos nas oficinas (Tabela 2), os mais citados foram: horários e dias escolhidos $(18,7 \%)$; pouco tempo para estudo individual (15,6\%); e deslocamento, carga horária extensa e falhas no convite para inscrição nas oficinas (12,5\% para cada item).

Quando questionados sobre os pontos positivos (Tabela 3), os mais citados foram: utilizar a própria ABE para ensinar $A B E(93,7 \%)$; qualidade dos artigos escolhidos para estudo prévio (87,5\%); e alta aplicabilidade prática $(81,2 \%)$.

Tabela1. Satisfação dos participantes acerca das oficinas realizadas.

\begin{tabular}{ccccccc}
\hline Participantes & Muito satisfatórias & Satisfatórias & Indiferentes & Insatisfatórias & Muito insatisfatórias & Total \\
\hline Resposta & $26(81,25 \%)$ & $6(18,75 \%)$ & 0 & 0 & 0 & 32 \\
Total & 26 & 6 & 0 & 0 & 32 \\
\hline
\end{tabular}

Tabela 2. Distribuição das respostas dos participantes em relação às dificuldades observadas nas atividades das oficinas.

\begin{tabular}{|c|c|c|c|}
\hline Quesito analisado & Sim & Não & Total \\
\hline Carga horária extensa & $4(12,5 \%)$ & $28(87,5 \%)$ & 32 \\
\hline $\begin{array}{l}\text { Pouco tempo para } \\
\text { estudo Individual }\end{array}$ & $5(15,625 \%)$ & $27(84,375 \%)$ & 32 \\
\hline $\begin{array}{l}\text { Artigos fornecidos } \\
\text { extensos ou } \\
\text { desinteressantes }\end{array}$ & 0 & $32(100 \%)$ & 32 \\
\hline Deslocamento & $4(12,5 \%)$ & $28(87,5 \%)$ & 32 \\
\hline Local de realização & $3(9,375 \%)$ & $29(90,625 \%)$ & 32 \\
\hline Explicação insatisfatória & 0 & $32(100 \%)$ & 32 \\
\hline $\begin{array}{l}\text { Pouca retenção de } \\
\text { conteúdo }\end{array}$ & 0 & $32(100 \%)$ & 32 \\
\hline $\begin{array}{l}\text { Dificuldade de } \\
\text { trabalhar em grupo }\end{array}$ & 0 & $32(100 \%)$ & 32 \\
\hline $\begin{array}{l}\text { Pouca aplicabilidade } \\
\text { prática }\end{array}$ & $1(3,125 \%)$ & $31(96,875 \%)$ & 32 \\
\hline $\begin{array}{l}\text { Questões mal } \\
\text { elaboradas no teste }\end{array}$ & 0 & $32(100 \%)$ & 32 \\
\hline $\begin{array}{l}\text { Horários e dias } \\
\text { escolhidos }\end{array}$ & $6(18,75 \%)$ & $26(81,25 \%)$ & 32 \\
\hline Ausência de intervalo & $1(3,125 \%)$ & $31(96,875 \%)$ & 32 \\
\hline $\begin{array}{l}\text { Convite para oficina } \\
\text { com falha de } \\
\text { comunicação }\end{array}$ & $4(12,5 \%)$ & $28(87,5 \%)$ & 32 \\
\hline
\end{tabular}

Tabela 3. Distribuição das respostas dos participantes em relação aos pontos positivos observados nas atividades das oficinas.

\begin{tabular}{|c|c|c|c|}
\hline Quesito analisado & Sim & Não & Total \\
\hline Carga horária adequada & $22(68,75 \%)$ & $10(31,25 \%)$ & 32 \\
\hline $\begin{array}{l}\text { Tempo suficiente para } \\
\text { estudo individual }\end{array}$ & $23(71,875 \%)$ & $9(28,125 \%)$ & 32 \\
\hline $\begin{array}{l}\text { Qualidade dos artigos } \\
\text { entregues }\end{array}$ & $28(87,5 \%)$ & $4(12,5 \%)$ & 32 \\
\hline Deslocamento & $7(21,875 \%)$ & $25(78,125 \%)$ & 32 \\
\hline Local de realização & $15(46,875 \%)$ & $17(53,125 \%)$ & 32 \\
\hline Explicação satisfatória & $25(78,125 \%)$ & $7(21,875 \%)$ & 32 \\
\hline $\begin{array}{l}\text { Adequada retenção de } \\
\text { conteúdo }\end{array}$ & $21(65,625 \%)$ & $11(34,375 \%)$ & 32 \\
\hline $\begin{array}{l}\text { Capacidade de } \\
\text { trabalhar em grupo }\end{array}$ & $24(75 \%)$ & $8(25 \%)$ & 32 \\
\hline $\begin{array}{l}\text { Alta aplicabilidade } \\
\text { prática }\end{array}$ & $26(81,25 \%)$ & $6(18,75 \%)$ & 32 \\
\hline $\begin{array}{l}\text { Questões bem } \\
\text { elaboradas no teste }\end{array}$ & $23(71,875 \%)$ & $9(28,125 \%)$ & 32 \\
\hline $\begin{array}{l}\text { Metodologia aplicada } \\
\text { usando a própria } A B E\end{array}$ & $30(93,75 \%)$ & $2(6,25 \%$ & 32 \\
\hline $\begin{array}{l}\text { Horário e dias } \\
\text { escolhidos }\end{array}$ & $18(56,25 \%)$ & $14(43,75 \%)$ & 32 \\
\hline
\end{tabular}


Tabela 4. Confiança e qualificação dos participantes após as oficinas para realizar atividades em ABE.

\begin{tabular}{cccccc}
\hline Participantes & Sim totalmente & Sim parcialmente & Não parcialmente & Não totalmente & Total \\
\hline Resposta & $12(37,5 \%)$ & $18(56,25 \%)$ & $1(3,125 \%)$ & $1(3,125 \%)$ & 32 \\
Total & $12(37,5 \%)$ & $18(56,25 \%)$ & $1(3,125 \%)$ & $1(3,125 \%)$ & 32 \\
\hline
\end{tabular}

Tabela 5. Avaliação da sensibilização dos docentes acerca dos benefícios da ABE no ensino-aprendizagem antes e depois das oficinas.

\begin{tabular}{ccccc}
\hline $\begin{array}{c}\text { Entende os benefícios da ABE no } \\
\text { ensino-aprendizagem? }\end{array}$ & Depois & & \\
\hline Antes & Não & Sim & Total & p-valor \\
\hline Não & $1(5,9 \%)$ & $16(94,1 \%)$ & $17(100 \%)$ & \\
Sim & $0(0 \%)$ & $15(100 \%)$ & $15(100 \%)$ & $0,0000^{*}$ \\
Total & $1(3,1 \%)$ & $32(96,9 \%)$ & $32(100 \%)$ & \\
\hline
\end{tabular}

*Teste de McNemar $(p<0,05)$.

Além disso, 93,7\% dos participantes referiram se sentir confiantes para conduzir atividades de $A B E$ em suas práticas diárias (Tabela 4).

Por fim, ressalta-se que $96,9 \%$ dos entrevistados referiram compreender os benefícios da $A B E$ após as oficinas realizadas e, destes, $53,1 \%$ não compreendiam anteriormente (Tabela 5). Essa diferença foi estatisticamente significante com $p=0,00$. Com base nesse resultado, podese afirmar que as oficinas, além de qualificarem os docentes, foram capazes de sensibilizá-los quanto aos benefícios no processo de ensino-aprendizagem.

\section{DISCUSSÃO}

Como a $\mathrm{ABE}$ visa desenvolver no estudante níveis avançados de aprendizagem (análise, aplicações, avaliações e criatividade), quatro aspectos são essenciais para alcançar esses objetivos: 1. equipes permanentes, estrategicamente formadas; 2. responsabilização dos alunos pela qualidade do trabalho individual e em equipe; 3. fornecimento de feedback frequente, imediato e oportuno; e 4. tarefas para a equipe que promovam tanto a aprendizagem individual como o desenvolvimento dela ${ }^{5,7}$.

Na elaboração das oficinas, todos esses aspectos foram considerados a fim de potencializar o aprendizado dos docentes participantes. Portanto, as equipes foram definidas de forma intencional levando em consideração características pessoais e profissionais, bem como a experiência prévia com ABE. Além disso, o condutor aplicou feedback constante durante todas as atividades e houve aumento progressivo de complexidade das tarefas propostas.

Cada equipe deveria ser constituída por pessoas com diferentes características, habilidades e personalidades ${ }^{5,7,17}$. A intenção era criar equipes cujos membros se comunicassem e soubessem negociar bem entre si, de forma que pudessem fazer contribuições equivalentes, atuar colaborativamente para atingir em conjunto os objetivos da capacitação e evitar situações de equipes disfuncionais, caracterizadas por disputas internas ou omissão na participação ${ }^{17}$.

Foi informado ainda aos participantes o caráter não avaliativo das oficinas, em que eles não seriam penalizados ou expostos. Enfatizou-se que se tratava de um momento de aprendizagem coletiva e que a participação de cada um era imprescindível para o sucesso de todos. Frame et al. ${ }^{18}$ ressaltam que um ambiente de honestidade e confiança entre os membros da equipe é fundamental para que todos se sintam à vontade para contribuir, acertar e errar; portanto, é uma grande responsabilidade do condutor garantir esse cenário.

Outra adaptação necessária em virtude do tempo disponível para as oficinas foi com relação ao número de questões do TGP. Aplicaram-se oito questões, número inferior ao preconizado por Michaelsen ${ }^{7}$ que sugere de dez a 20 perguntas. Contudo, percebeu-se que o número de questões não interferiu no desempenho final das equipes, provavelmente porque o conteúdo era bem delimitado e os objetivos educacionais puderam ser contemplados.

Ainda sobre a importância da qualificação docente, Nation et al. ${ }^{19}$, ao compararem a satisfação e o desempenho de alunos em duas escolas de farmácia no Reino Unido, apontaram que os resultados foram mais positivos naquela com mais professores capacitados para executar a metodologia da ABE.

De acordo com Gullo et al. ${ }^{20}$, como os professores/ instrutores desempenham um papel fundamental na $A B E$, eles precisam ser especificamente treinados sobre essa estratégia educacional antes de aplicá-la. Para Yang et al. ${ }^{21}$, esse treinamento deve ter três objetivos: 1. ensinar a desenvolver 
questões apropriadas que se aproximem da prática clínica com dificuldade adequada; 2. facilitar a aprendizagem dos alunos, encorajando-os e ofertando amplas opções de práticas para desenvolvimento de suas habilidades profissionais; e 3. dar treinamento sobre o feedback imediato e oportuno com técnicas corretas.

Contudo, não foi encontrado na literatura nenhum estudo que aborde a qualificação de docentes de Medicina em atividades de $A B E$, sendo esse o principal fator que motivou a realização da presente pesquisa. Espera-se com isso contribuir para o desenvolvimento dos professores das mais diversas instituições de ensino. Diversos autores ${ }^{22-24}$ destacam ainda que a capacitação docente deve prepará-los para que possam atuar em um ambiente mais barulhento, dinâmico e menos previsível, além de desencorajar posturas autoritárias e que não abrem espaço para o diálogo e a construção coletiva do conhecimento.

Quando se avaliou a satisfação dos participantes com relação à metodologia proposta nas oficinas, percebeu-se que $81,2 \%$ atribuíram "satisfação máxima" (5 na escala Likert) e os demais a classificaram como "satisfatória". Dessa forma, todos os docentes aprovaram a técnica empregada. Tais achados concordam com Haj-Ali et al. ${ }^{25}$ que, em seu estudo nos Emirados Árabes Unidos, perceberam que a pontuação em exames de alunos submetidos à $\mathrm{ABE}$ foi superior à daqueles que participaram de atividades de ensino tradicional; além disso, $72 \%$ afirmaram que estavam satisfeitos com as atividades de $A B E$ e outros $22 \%$ responderam que a satisfação era parcial.

Outros autores apontam ainda que participantes de atividades de $A B E$ recomendam fortemente que elas sejam expandidas e aplicadas a outras disciplinas, módulos ou cursos ${ }^{26}$, além de perceberem que o entusiasmo com essa estratégia educacional não diminui com o passar do tempo 9,24,27,28.

Santana et al. ${ }^{29}$ descreveram um curso de Genética Médica organizado em cinco blocos de assuntos, compreendendo 25 doenças genéticas, nos quais uma sessão de $A B E$ foi conduzida em cada um desses blocos. Participaram um total de 290 alunos, dos quais $96 \%$ se posicionaram satisfeitos com o método de ABE. Além disso, 97\% dos estudantes pontuaram que a $A B E$ os ajudou a aprender e $87 \%$ aprovariam o uso da metodologia em outras etapas de seu curso de Medicina.

Quando indagados sobre os pontos negativos nas oficinas, os mais citados foram: horários e dias escolhidos e pouco tempo para estudo individual. No geral, percebeu-se que poucos docentes relataram pontos negativos e, os que os mencionaram, destacaram que as oficinas foram realizadas fora da carga horária dedicada à instituição e em uma unidade diferente do curso de Medicina, além de considerarem sete dias um período curto para o estudo individual.

Esses achados corroboram os resultados obtidos por Allen et al. ${ }^{30}$ cujo trabalho pontuou que os principais fatores que levavam professores a não adotar a $A B E$ eram localizações distantes, resistência em mudar a forma de ensinar e falta de tempo hábil para treinamento. Em estudo de Silva Junior et al. ${ }^{15}$, a necessidade de estudo prévio fora de sala de aula foi apontada como o principal dificultador.

Todavia, as respostas diferem do observado por Balwan et al. ${ }^{31}$ em estudo realizado com médicos residentes em Nova York, que destacaram como fragilidades: a qualidade dos artigos selecionados para estudo prévio, os objetivos propostos e a má elaboração das questões do teste escrito. Essas variáveis não foram referidas pelos professores do presente estudo, cujos artigos e questões foram bem avaliados pelos participantes.

Quando questionados sobre os pontos positivos, os mais citados foram: utilizar a própria $A B E$ para ensinar $A B E$, qualidade dos artigos escolhidos para estudo prévio e alta aplicabilidade prática. Dessa forma, esse resultado corrobora a hipótese de que a tecnologia educacional desenvolvida, que utiliza oficinas de $A B E$ para falar sobre $A B E$, atraiu a atenção dos participantes e permitiu maior interesse e sensibilização sobre o método.

Altintas et al. ${ }^{32}$, ao avaliarem alunos do quinto ano de Medicina na disciplina de Oftalmologia, identificaram que a maioria dos participantes considerou a atividade de ABE proposta muito boa para atingir os objetivos das aulas, possibilitou melhor entendimento, foi mais interessante, garantiu maior participação dos discentes e os envolveu mais em todo o processo quando essa metodologia foi comparada às aulas tradicionais, resultado semelhante ao obtido pelo presente estudo.

Destaca-se ainda que a aplicabilidade prática foi um dos itens mais mencionados. Quando se pensou sobre o formato e as tecnologias a serem utilizadas nas oficinas, inicialmente havia a intenção de utilizar aplicativos ou a plataforma on-line. Contudo, posteriormente se decidiu por fazer as oficinas com os materiais mais acessíveis e menos dispendiosos possíveis, a fim de demonstrar aos participantes que não eram necessários grandes investimentos para realizar atividades pontuais de $A B E$. Esta se mostrou uma escolha acertada, conforme a resposta dos questionários avaliativos.

Além disso, 93,7\% dos participantes referiram se sentir confiantes para conduzir atividades de ABE em suas práticas diárias. Esses achados concordam com Jost et al. ${ }^{33}$, que perceberam que a $A B E$ foi atrativa para alunos e professores, considerada relativamente fácil de aprender e, em seguida, ser implementada, e com Allen et al. ${ }^{30}$, que constataram que $72 \%$ dos docentes que começam a empregar a $A B E$ decidem por mantê-la em suas práticas. 
Porfim, ressalta-se que $96,9 \%$ dos entrevistados referiram compreender os benefícios da ABE após as oficinas realizadas, e, destes, 53,1\% não compreendiam anteriormente, o que permite afirmar que as oficinas, além de qualificarem os docentes, foram capazes de sensibilizá-los quanto aos benefícios dessa estratégia educacional no processo de ensino-aprendizagem. Esses dados concordam com estudos realizados em cursos de Farmácia nos Estados Unidos, cujos resultados demonstraram que, apesar das dificuldades, os docentes percebem que a $A B E$ incrementa o engajamento e o aprendizado dos alunos ${ }^{30}$.

Dessa forma, percebe-se que o modelo proposto de oficinas de qualificação docente em $A B E$ se mostrou uma ferramenta com bons resultados em incremento de conhecimento e sensibilização quanto aos seus benefícios, e ainda com grande potencial de desenvolvimento e aperfeiçoamento para que melhor se adapte às realidades locais sem que haja prejuízo ao processo de ensino-aprendizagem.

\section{CONCLUSÃO}

Relatou-se, neste estudo, um modelo de oficina de qualificação docente em $A B E$, assim como foram descritas as avaliações feitas pelos participantes acerca da metodologia empregada, cujos resultados apontam para o sucesso das oficinas, uma vez que os participantes relataram a possibilidade de aplicar essa estratégia de ensino com maior segurança e assertividade em suas rotinas diárias. Constatou-se que a tecnologia educacional desenvolvida é uma ferramenta válida para replicabilidade em outras instituições de ensino que desejem implementá-la em suas matrizes curriculares.

Espera-se que este trabalho possa contribuir para a aprendizagem sobre essa estratégia educacional e que possa descomplicar e tornar acessível a sua aplicação, a fim de estimular docentes e instituições não familiarizados com a $\mathrm{ABE}$ a introduzir essa valiosa ferramenta em suas realidades.

Outrossim, estudos futuros podem ser realizados para conhecer a realidade de aplicação da ABE em outras instituições de ensino públicas ou privadas e em outros cursos das diversas áreas, assim como propor o desenvolvimento de novas tecnologias educacionais baseadas na $A B E$ que podem ser elaboradas com vistas a melhorar a qualidade do ensino em saúde.

\section{CONTRIBUIÇÃO DOS AUTORES}

Mário Roberto Tavares Cardoso de Albuquerque participou da concepção e do desenvolvimento do estudo (desde o objeto a ser investigado até a criação da hipótese), do desenho metodológico (planejamento dos métodos para gerar os resultados), da supervisão (responsável pela organização e execução do projeto e pela escrita do manuscrito), da coleta, do tratamento e da organização dos dados, da análise/ interpretação dos resultados, do levantamento da literatura (participou da pesquisa bibliográfica e do levantamento de artigos) e da redação e revisão do texto final. Nara Macedo Botelho e Milena Coelho Fernandes Caldato participaram da supervisão do estudo (foram responsáveis pela organização e execução do projeto e pela escrita do manuscrito) e da revisão crítica do texto (revisaram o conteúdo intelectual do manuscrito antes da apresentação final).

\section{CONFLITO DE INTERESSES}

Declaramos não haver conflito de interesses.

\section{FINANCIAMENTO}

Declaramos não haver financiamento.

\section{REFERÊNCIAS}

1. Bleske BE, Remington TL, Wells TD, Klein KC, Guthrie SK, Tingen JM, et al. A randomized crossover comparison of Team-Based Learning and lecture format on learning outcomes. Am J Pharm Educ. 2016;80(7):120-4.

2. Remington TL, Bleske BE, Bartholomew T, Dorsch MP, Guthrie SK, Klein KC et al. Qualitative analysis of student perceptions comparing Team-Based Learning and traditional lecture in a pharmacotherapeutics course. Am J Pharm Educ. 2017;81(3):55-63.

3. Searle NS, Haidet P, Kelly PA, Schneider VF, Seidel CL, Richards BF. Team learning in medical education: initial experiences at ten institutions. Acad Med. 2003;78(10):S55-S8.

4. Burgess A, Ayton T, Mellis C. Implementation of team-based learning in year 1 of a PBL based medical program: a pilot study. BMC Med Educ. 2016;16:49-55.

5. Michaelsen LK, Sweet M. Fundamental principles and practices of TeamBased Learning. In: Michaelsen LK, Parmelee D, MacMahon KK, Levine RE, editors. Team-Based Learning for health professions education: a guide to using small groups for improving learning. Sterling: Stylus; 2008. p. 9-34.

6. Krug RR, Vieira MSM, Maciel MVA, Erdmann TR, Vieira FCF, Koch MC, et al et al. O "bê-á-bá" da Aprendizagem Baseada em Equipe. Rev Bras Educ Med. 2016;40(4):602-10.

7. Michaelsen LK. Getting started with Team-Based Learning. In: Michaelsen LK, Knight $A B$, Dee $F L$, editors. A transformative use of small groups. Westport: Praeger; 2002. p. 27-52.

8. Michaelsen LK, Parmelee DX, McMahon LL. Team-Based Learning for health professions education. A guide to using small groups for improving learning. Sterling: Stylus; 2008.

9. Gray J, Fana GT, Campbell TB, Hakim JG, Borok MZ, Aagaard EM. Feasibility and sustainability of an interactive team-based learning method for medical education during a severe faculty shortage in Zimbabwe. BMC Med Educ. 2014;14:63-7.

10. Bleske BE, Remington TL, Wells TD, Dorsch MP, Guthrie SK, Stumpf JL, et al. Team-based learning to improve learning outcomes in a therapeutics course sequence. Am J Pharm Educ. 2014;78(1):13-7.

11. Parmelee $D$, Michaelsen LK. Twelve tips for doing effective Team-Based Learning (TBL). Med Teach. 2012;32:118-22.

12. Reimschisel $T$, Herring $A L$, Huang J, Minor TJ. A systematic review of the published literature on team-based learning in health professions education. Med Teach. 2017;39(12):1227-37.

13. Thiollent M. Metodologia da pesquisa-ação. 16a ed. São Paulo: Cortez; 2013.

14. Lima VV, Gomes R, Padilha RQ, Oliveira MS, Tempski PZ. Aprendizagem baseada em equipes: diretrizes, etapas e recomendações. Nota técnica $\mathrm{n}^{\circ}$ 4. São Paulo: Instituto Sírio-Libanês de Ensino e Pesquisa; 2016. 
15. Silva Junior GB, Medeiros MAS, Oliveira JGR, Catrib AMF, Jardim MHAG. Team-Based Learning: successful experience in a public health graduate program. Rev Bras Educ Med. 2017;41(3):397-401.

16. Team-Based Learning Collaborative [acesso em 10 jan 2020]. Disponível em: https://www.teambasedlearning.org.

17. Farland MZ, Sicat BL, Franks AS, Pater KS, Medina MS, Persky AM. Best practices for implementing Team-Based Learning in Pharmacy Education. Am J Pharm Educ. 2013;77(8):177-86.

18. Frame TR, Cailor SM, Gryka RJ, Chen AM, Kiersma ME, Sheppard L. Student perceptions of Team-based Learning vs Traditional Lecture-based Learning. Am J Pharm Educ. 2015; 79(4):51-61.

19. Nation LM, Tweddell S, Rutter P. The applicability of a validated teambased learning student assessment instrument to assess United Kingdom pharmacy students' attitude toward team-based learning. J Educ Eval Health Prof. 2016;13:30-4.

20. Gullo $\mathrm{C}, \mathrm{Ha} \mathrm{TC}$, Cook $\mathrm{S}$. Twelve tips for facilitating team-based learning. Med Teach. 2015;37(9):819-24.

21. Yang L, Jiang L, Xu B, Liu SQ, Liang YR, Ye JH, et al. Evaluating team-based, lecture-based, and hybrid learning methods for neurology clerkship in China: a method-comparison study. BMC Med Educ. 2014;14(3):98-104.

22. Kibble JD, Bellew C, Asmar A, Barkley L. Team-based learning in large enrollment classes. Adv Physiol Educ. 2016;40(4):435-42.

23. Franks AS. Enhancing Team-Based Active Learning through handson experience with nicotine replacement therapy. Am J Pharm Educ. 2013;77(6):128-33.

24. Branney J, Priego-Hernández J. A mixed methods evaluation of teambased learning for applied pathophysiology in undergraduate nursing education. Nurse Educ Today. 2018;61:127-33.
25. Haj-Ali R, Al Quran F. Team-based learning in a preclinical removable denture prosthesis module in a United Arab Emirates dental school. J Dent Educ. 2013;77(3):351-7.

26. Burgess AW, Mellis C, McGregor DM. Applying established guidelines to team-based learning programs in medical schools: a systematic review. Acad Med. 2014;89:678-88.

27. Masocatto NO, Couto WJ, Matta TS, Porfirio GJM. Percepção de alunos de curso de graduação em Medicina sobre o Team-Based Learning (TBL). Rev Bras Educ Med. 2019;43(3):111-4.

28. Reyes-Torres GH, Saavedra J, Aguayo-Vergara M. Aprendizaje basado en equipos en un curso de Ingeniería en Educación Superior. Educación. 2020;44(1):257-76.

29. Santana VC, Oliveira CR, Ramos RB. First-year students' perceptions of team-based learning in a new medical genetics course. Rev Bras Educ Med. 2019;43(3):170-7.

30. Allen RE, Copeland J, Franks AS, Karimi RZ, McCollum M, Riese II DJ, et al. Team-Based Learning in US colleges and schools of Pharmacy. Am J Pharm Educ. 2013;77(6):115-23.

31. Balwan S, Fornari A, DiMarzio P, Verbsky J, Pekmezaris R, Stein J, et al. Use of Team-Based Learning Pedagogy for Internal Medicine Ambulatory Resident Teaching. J Grad Med Educ. 2015;7(4):643-8.

32. Altintas L, Altintas O, Caglar Y. Modified use of team-based learning in an ophthalmology course for fifth-year medical students. Adv Physiol Educ. 2014;38(1):46-8

33. Jost $M$, Brüstle P, Giesler M, Rijntjes M, Brich J. Effects of additional teambased learning on students' clinical reasoning skills: a pilot study. BMC Res Notes. 2017;10(1): 282-8. 